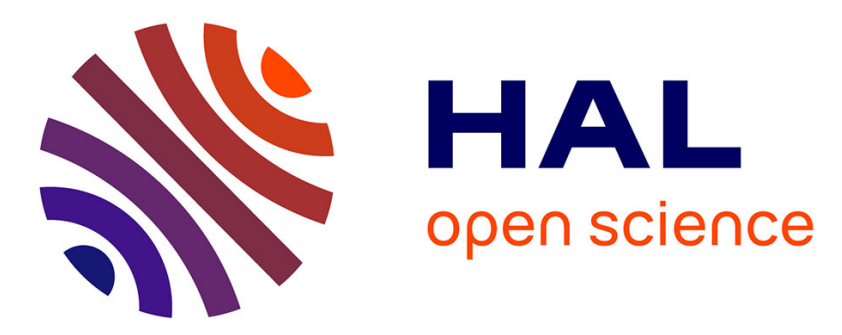

\title{
Assembly Process Design: Performance Evaluation Under Ergonomics Consideration Using Several Robot Collaboration Modes
}

Anthony Quenehen, Stephane Thiery, Nathalie Klement, Lionel Roucoules, Olivier Gibaru

\section{To cite this version:}

Anthony Quenehen, Stephane Thiery, Nathalie Klement, Lionel Roucoules, Olivier Gibaru. Assembly Process Design: Performance Evaluation Under Ergonomics Consideration Using Several Robot Collaboration Modes. IFIP International Conference on Advances in Production Management Systems APMS 2020: Advances in Production Management Systems. Towards Smart and Digital Manufacturing, Aug 2020, Novi Sad, France. pp.477-484, 10.1007/978-3-030-57997-5_55 . hal-03030674

\section{HAL Id: hal-03030674 https://hal.science/hal-03030674}

Submitted on 30 Nov 2020

HAL is a multi-disciplinary open access archive for the deposit and dissemination of scientific research documents, whether they are published or not. The documents may come from teaching and research institutions in France or abroad, or from public or private research centers.
L'archive ouverte pluridisciplinaire HAL, est destinée au dépôt et à la diffusion de documents scientifiques de niveau recherche, publiés ou non, émanant des établissements d'enseignement et de recherche français ou étrangers, des laboratoires publics ou privés. 


\title{
Assembly process design: performance evaluation under ergonomics consideration using several robot collaboration modes
}

\author{
Anthony Quenehen, Stephane Thiery, Nathalie Klement, Lionel Roucoules, and \\ Olivier Gibaru
}

Arts et Métiers Institute of Technology, LISPEN, HESAM Université, F-59000 Lille, France

\begin{abstract}
This paper aims at studying the combination of different collaboration modes between operator and collaborative robot in order to optimize an assembly process for both economic and ergonomic objectives. Based on a real case study, and using a energy expenditure ergonomic model, the authors have determined by experiment the different ergonomic and economic variables under each possible collaboration mode. They propose a set of indicators to evaluate the quality of assignment solutions, as well as a multi-objective cost function to determine optimal trade-offs between the different collaboration modes. An initial set of trials has indicated that combining several modes of collaboration may deliver benefits for both economic and ergonomic performance.
\end{abstract}

Keywords: Collaborative robotics · Process design · Ergonomics performance · Collaboration modes · Optimization.

\section{Introduction}

Collaborative robotics has opened new ways for introduction of automation into manual processes. Indeed, the opportunity for human and collaborative robot (cobot) of sharing the same environment without physical safety device (under certain circumstances) has made their interaction potentially smoother. This may lead to design a collaborative (human and cobot) process using work balancing approach. Besides their inherent limitations in handling complex assembly tasks, most cobots have limited payload and speed compared to industrial robots. This guarantees safe interaction with operators, but hinders cobots economic competitiveness. Nevertheless, their ability to execute or assist human tasks delivers solutions for process ergonomics improvement, thus representing a competitive asset when it comes to balance both economic and ergonomic performance, opening way for repetitive stress injuries reduction and / or management of disabled workforce within the production environment. 


\section{Related works}

Several types of safe interaction can be foreseen in the context of assembly operations, ranging from physical separation between operator and cobot to simultaneous action on the same work piece. Thiemermann [9] proposes a classification based on spatial and temporal separation, highlighting four interaction categories, see Fig. 1. In the process of designing a collaborative assembly process, we propose to focus on the 'synchronisation' and 'cooperation' categories, which are specific to cobot. Synchronisation enables to handle the task allocation as a balancing problem under constraints. Usual constraints may be precedence of tasks, resource availability, along with potential for automation of task based on technical feasibility factors [4], [5], [10]. As a result of this decision process, task may be allocated to either operator or cobot. Cooperation category follows a similar decision process, offering an additional allocation option towards 'operator and cobot', hence using both resources for the concerned task [1]. In both cases, proposed solutions are evaluated against performance metrics, as Cycle Time (CT) or makespan [7] for instance.

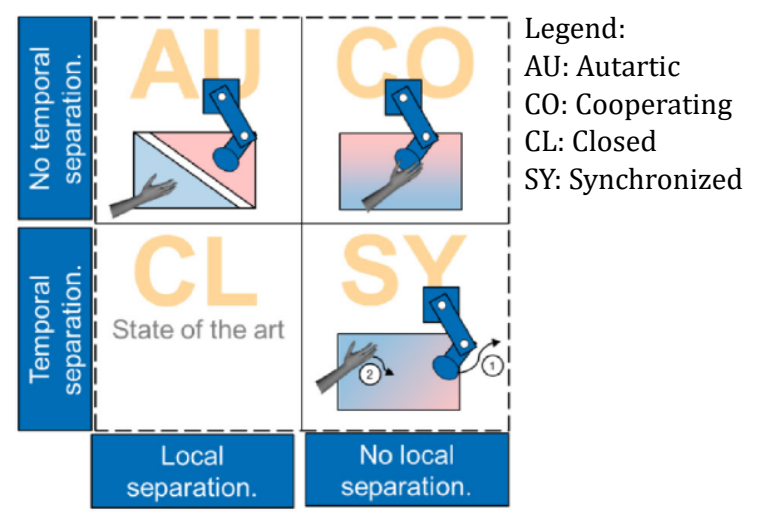

Fig. 1. Human robot interaction categories, adapted from [9]

Ergonomic improvement, also representing a potential benefit of cobot usage, has already received attention from researchers. The subsequent trade offs between economical performance and ergonomic improvements have been studied in the form of multi objective optimization, based on applicable ergonomic models [7], [11]. Nevertheless, this has been done under the assumption of task allocation towards either operator or cobot.

Several ergonomic models, such as RULA (Rapid Upper Limb Assessment) [6], are available to assess and quantify the strain generated by posture, efforts and movements throughout the execution of process tasks. Additional approaches based on energy expenditure propose more quantitative evaluation and 
results [3]. They also express the ergonomic load as a necessary relaxation time (RT), required when the energy spent during the task exceeds a given threshold value [2]. Based on formulas related to each type of movement, they can offer quick calculation of the energy expenditure for each work element. More interestingly, they express the ergonomic cost (relaxation time) in the same dimension as the economic cost (cycle time), providing more realistic view of the trade off between the two objectives. For these reasons we will select the PMES (Predetermined Motion Energy System) model proposed by [2], and give further details in following section.

Building on existing work, we propose to design a process considering a three way trade off between operator, cobot, or joint action of operator and cobot for each task (cooperative mode). Indeed, allocating task to cobot whose motion speed is slow will improve ergonomic performance at the expense of an increased task time (TT), as using cooperation mode will deliver less ergonomic improvement, but without impacting TT. Nevertheless, both resources will be required for a single task in that case. Our motivation is to enlarge the field of possible solutions, since each option dominates the other two (see Table 1) on one of the criteria of the problem (economic performance, ergonomic, resource mobilisation). Additionally, we propose to ground our work on an experimental case study, as we deem important to test the relevancy and applicability of the proposed solutions, specially when the supporting model complexity has been increased.

Table 1. A qualitative comparison of collaboration modes under performance criteria

\begin{tabular}{lccc}
\hline & \multicolumn{3}{c}{ Operator Cobot Cooperation } \\
\hline $\begin{array}{l}\text { Eco. } \\
\text { performance }\end{array}$ & High & Low & High \\
\hline $\begin{array}{l}\text { Ergo. } \\
\text { performance }\end{array}$ & Low & High & Intermediate \\
$\begin{array}{l}\text { Parallelisation } \\
\text { of tasks }\end{array}$ & High & High & Low \\
\hline
\end{tabular}

\section{Proposed problem modelisation}

Assignment notations. We note $t=0$ the starting time of the process cycle, and represent an assignment solution $S$ by $\left[\left[t_{1}, A_{1}\right],\left[t_{2}, A_{2}\right], \ldots\left[t_{N}, A_{N}\right]\right]$, where $N$ notes the number of tasks, $t_{i}$ the starting time of task $i$, and $A_{i} \in$ $\{$ '10','01','11'\} its allocation (respectively '10' to an operator, '01' to cobot, and '11' to both in cooperation mode). An assignment solution $S$ provides operator's and cobot's respective cycles times $C T_{o}(S)$ and $C T_{c}(S)$ (defined as "the time 
when the operator, resp the cobot, finishes his last task"), and the required relaxation time $R_{t o t}(S)$ for the operator.

Cost function. We define an economic cost $C_{\text {eco }}(S)$ as equal to the process cycle time. Assuming that in case of idle time between two tasks, operator will rather slow down his pace to fill this gap with continuous work than stopping and relaxing, we consider that relaxation can only happen after operator's cycle is completed. Thus, we define an ergonomic $\operatorname{cost} C_{\text {ergo }}(S)$ as the remaining amount of relaxation time after cycle completion (in case of the operator finishes first i.e. $C T_{c}(S)-C T_{o}(S)>0$, he can start relaxing while the cobot finishes the process cycle, otherwise this cost is $\left.R_{t o t}(S)\right)$. This leads to the following cost expressions:

$$
\left\{\begin{array}{c}
C_{\text {eco }}(S)=\max \left\{C T_{o}(S), C T_{c}(S)\right\} \\
C_{\text {ergo }}(S)=\max \left\{0, R_{\text {tot }}(S)-\left(C_{\text {eco }}(S)-C T_{o}(S)\right)\right\}
\end{array}\right.
$$

Economic cost and ergonomic cost being expressed in the same unit (time in second), we can easily design a cost function. As part of an upcoming work, we aim at finding assignment solutions $S$ minimizing the following cost function:

$$
J_{\alpha}(S)=(1-\alpha) \cdot C_{e c o}(S)+\alpha \cdot C_{\text {ergo }}(S)
$$

where $\alpha \in[0,1[$ stands for a given trade-off between ergonomic and economic cost. The apparent complexity to solve this problem by a solver, as an optimization problem under constraints (precedence and resources availability), points towards an evolutionary algorithm based solutions. We plan to use genetic algorithm or reinforcement learning.

Key Performance Indicators. To assess the performances of an assignment solution $S$, we output the followings economic and ergonomic indicators:

$$
I_{\text {eco }}(S)=\frac{C_{\text {ref }}}{C_{\text {eco }}(S)} \text { and } I_{\text {ergo }}(S)=\min \left\{\frac{C_{\text {eco }}(S)}{C T_{o}(S)+R_{\text {tot }}(S)}, 1\right\}
$$

with $C_{r e f}$ representing the cycle time of a full manual process. The higher $I_{e c o}$, the better the economic performance. Compared to a full manual process, cooperative mode assignment would enable to significantly reduce the ergonomic cost, but also to marginally reduce the cycle time. Therefore, achieving $I_{\text {eco }}>1$ is possible. Note that an upper bound exists, corresponding to perfect parallelisation of tasks between operator and cobot, assuming compliance to all precedence and feasibility constraints are achieved. For example, if the cobot is $k$ times slower than operator, this bound is $I_{e c o}=1+1 / k$. Further work will determine applicable maximum values for $I_{\text {eco }}$ (setting $\alpha=0$ in Eq.2). Concerning $I_{\text {ergo }}$, the closest to 1 , the best the ergonomic performance is. Indeed $I_{\text {ergo }}$ inferior to 1 means a remaining relaxation time is still required after completion of the process. If $C_{\text {eco }}(S) /\left(C T_{o}(S)+R_{t o t}(S)\right)>1$, operator finishes to relax before end of process cycle. From ergonomic point of view, this situation does not represent 
any additional benefit (only inefficiency of resource usage). Therefore, as per Eq.3, $I_{\text {ergo }}$ maximum value has been set to 1 .

Also, as a sub KPI, we compute the resource usage ratio as percentage of the cycle time, where $I d l e_{o}$ and $I d l e_{c}$ are the respective idle times of the operator and the cobot during the cycle:

$$
\left\{\begin{array}{l}
U_{o}(S)=\frac{C_{e c o}(S)-I d l e_{o}(S)+\min \left\{C_{e c o}(S)-C T_{o}(S), R_{t o t}(S)\right\}}{C_{e c o}(S)} \times 100 \\
U_{c}(S)=\frac{C_{e c o}(S)-I d l e_{c}(S)}{C_{e c o}(S)} \times 100
\end{array}\right.
$$

Indeed, when the cobot finishes last, idle time at the end of operator's cycle can be used for relaxation.

\section{Application: problem setting}

In order to support this approach, the assembly process of a pneumatic cylinder has been selected as a case study. Offering different types of operations (picking, setting, inserting, screwing), with various levels of complexity, it may give relevance to the potential for automation. The simple geometric nature of most sub components may also permit relatively simple handling operation from the cobot with standard effectors.

This assembly process is divided in fourteen tasks, with a limited number of precedence constraints (see Fig. 2). Thus, it should offer a large number of possibilities for parallelisation of tasks, and open sufficient areas for solutions to understand the potential and limitations of the proposed model.

The description of the manual assembly process can be seen on the following URL: bit.ly/2w8Iwf0. Based on the video analysis of several cycles of the assembly process, repeatable time values have been determined for each work element of every task, in order to populate the model. Likewise, each work element has been assessed through PMES to calculate its relative energy expenditure, using the formulas proposed in [3], and determine related relaxation time if applicable. In order to populate each possible task execution mode (operator, cobot, or cooperative), two versions of the process have been run and analyzed: fully manual version (100\% of tasks done by operator), and a version where cooperation mode has been maximized to re-calculate TT and RT. Cobot related TT have been determined by applying a coefficient of $k=5.9$ on the operator TT, based on previous work in the cobot usage for similar case study [8]. When task could not be allocated to cobot due to its complexity (i.e. bolt tightening), or cooperation mode is irrelevant (i.e task consists in moving a single piece), related TT and / or RT have been set to the $\infty$ value, to exclude them from potential selection based on a cost function. Overall results are presented in Fig. 2 . 


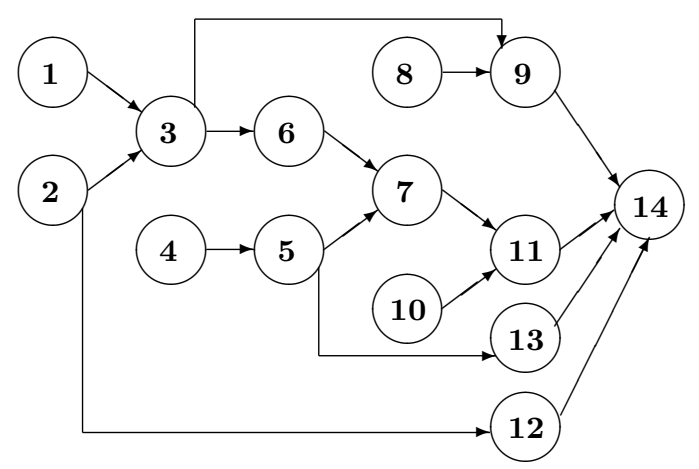

\begin{tabular}{r|c|c|c} 
& $\begin{array}{c}\text { Human } \\
\text { TT/RT }\end{array}$ & $\begin{array}{c}\text { Cobot } \\
\text { TT }\end{array}$ & $\begin{array}{c}\text { Coop. } \\
\text { TT/RT }\end{array}$ \\
\hline 1 & $1.2 / 1$ & 6.8 & $\infty / \infty$ \\
2 & $2.3 / 2.2$ & 13.6 & $\infty / \infty$ \\
3 & $14.3 / 9.7$ & $\infty$ & $12.3 / 1.5$ \\
4 & $1.5 / 0.2$ & 8.9 & $1 / 0.3$ \\
5 & $2.1 / 1.6$ & 12.4 & $1.8 / 0.4$ \\
6 & $2 / 1.2$ & 11.8 & $1.8 / 0.4$ \\
7 & $14.3 / 9.7$ & $\infty$ & $12.3 / 1.5$ \\
8 & $10.2 / 3.4$ & 60.2 & $10 / 2$ \\
9 & $11 / 7.3$ & 64.9 & $11 / 3$ \\
10 & $10.2 / 12.8$ & 60.2 & $10 / 2$ \\
11 & $11 / 17$ & 64.9 & $11 / 3$ \\
12 & $3.5 / 2.4$ & $\infty$ & $3.5 / 0.6$ \\
13 & $3.5 / 2.4$ & $\infty$ & $3.5 / 0.6$ \\
14 & $1.3 / 1.9$ & 7.7 & $\infty / \infty$
\end{tabular}

Fig. 2. Precedence graph of our problem, with Task Time (TT) and Relaxation Time (RT) in seconds for each type of task assignment.

\section{Identification of solution areas}

Initial trials were done by maximizing utilisation of each specific collaboration mode (operator, cobot, cooperation), in compliance with precedence constraints, but without investigating potential benefit of altering assembly sequence at this stage. Thus, we considered the following four specific assignment solutions:

- $S_{\text {manual }}$ full manual process. Each task is allocated to the operator.

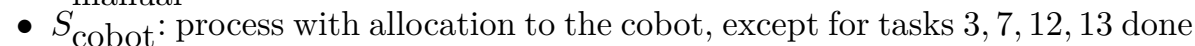
in parallel by the operator the earliest possible (no usage of cooperation mode).

- $S_{\text {coop-eco }}\left(\right.$ resp $S_{\text {coop-ergo }}$ ): process with highest usage of cooperation mode. Tasks 1, 2, 14, not eligible for cooperation, are assigned to the operator (resp the cobot) to obtain the best economic (resp ergonomic) solution under maximum cooperation mode.

Table 2 shows costs and KPIs of these solutions, and Fig. 3 visualises their relative position in the 2D-space of ergonomic and economic performances. In Fig. 3, KPIs obtained for the six others maximum cooperation modes (there is $2^{3}$ combinations of tasks assignments $1,2,14$ to operator or cobot) are also represented to show that, indeed, they are located in the square area delimited by KPIs of the best economic and best ergonomic under maximum cooperation mode. These first trials were done to visualize the KPI behaviours under maximum usage of each allocation mode. As expected, none of them is optimum in terms of economic and ergonomic performance (maximum cobot usage is one leading to ergonomic KPI of 1 , at the expense of a severe KPI economic loss).

Appropriate combination of the different modes will deliver higher level of both economic and ergonomic performance. The upcoming algorithm development will aim at characterising such KPI solution areas, and their borders for a same fixed trade-off $\alpha$. Resolution will be done respectively with and without the 
Table 2. Costs and KPIs of initial trials

\begin{tabular}{|c|c|c|c|c|c|c|c|c|c|}
\hline & & & & \multicolumn{2}{|c|}{ Costs (Eq.1) } & \multicolumn{2}{|c|}{ KPI (Eq.3) } & \multicolumn{2}{|c|}{ Usage (Eq. } \\
\hline Solution & $C T_{o}$ & $R_{\text {tot }}$ & $C T_{c}$ & $C_{\text {eco }}$ & $C_{\text {ergo }}$ & $I_{\text {eco }}$ & $I_{\text {ergo }}$ & $U_{o}$ & $U_{c}$ \\
\hline$S_{\text {manual }}$ & 88.4 & 72.8 & 0 & 88.4 & 72.8 & 1 & 0.548 & $100 \%$ & $0 \%$ \\
\hline$S_{\text {cobot }}$ & 67.8 & 24.2 & 311.4 & 311.4 & 0 & 0.284 & 1 & $19.2 \%$ & $100 \%$ \\
\hline$S_{\text {coop-eco }}$ & 83 & 20.4 & 81.7 & 83 & 20.4 & 1.065 & 0.803 & $100 \%$ & $94.22 \%$ \\
\hline$S_{\text {coop-ergo }}$ & 98.6 & 15.3 & 106.3 & 106.3 & 7.6 & 0.832 & 0.933 & $80.81 \%$ & $100 \%$ \\
\hline
\end{tabular}

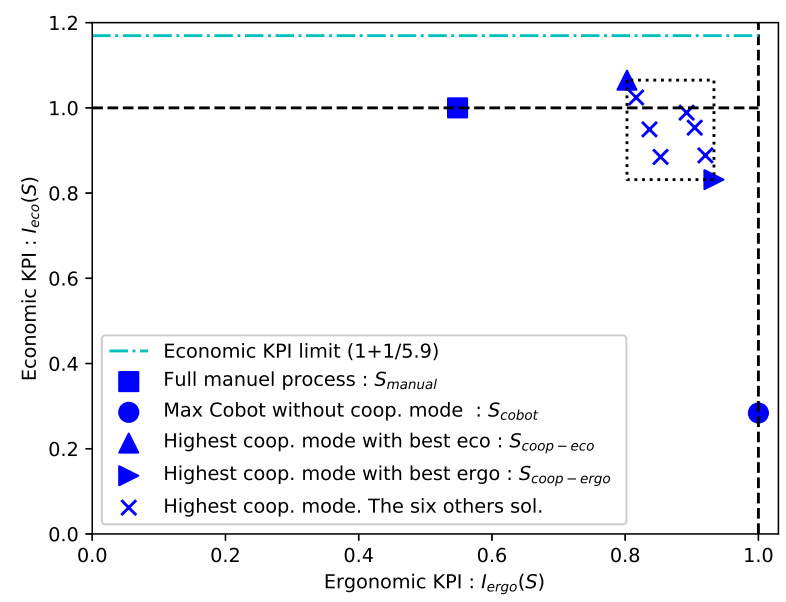

Fig. 3. Relative KPI's position of the different initial trials.

cooperation mode. By comparing respective solutions, we will be in a position to assess the additional benefits of the cooperative mode.

In order to assess the validity of the proposed model and results the solutions

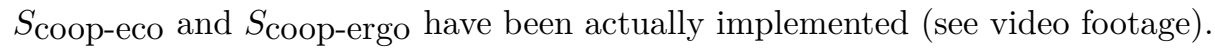
Despite minor discrepancies in task times when recombining them into new processes, it appeared that a significant factor is the possible mismatch between the end condition of a task and start condition of the next depending on their respective collaboration mode. This leads to adjust the content of the concerned tasks, impacting their TT. Modeling and populating such phenomenon would increase significantly problem complexity, for potential limited benefit in prediction accuracy. Besides, as per regular automation approach, continuous improvement loops may be applied to several tasks involving cobot (trajectory optimization), improving further the economic performance. Furthermore, as it can be seen on the process video footage, the cyclic nature of the production enables to parallelise the last tasks of an instance and the first tasks of the next. This factor is 
not reflected in the current problem setting and will require updating $C_{\text {eco }}$ and $C_{\text {ergo }}$ expressions.

\section{Conclusion and further work}

In this article, we have proposed an analysis of both economic and ergonomic KPIs through the combination of several collaboration modes between operator and cobot. A part of upcoming work will be the resolution of our optimization problem based on the designed cost function, using genetic algorithm or reinforcement learning. This will support a more accurate definition of solutions areas, and offer more quantitative evaluations of additional benefits brought by the combination of the different collaboration modes. Additionally, experimental phase has highlighted valuable elements that may benefit to the initial model, and open way to study a comprehensive process design approach, based on an iterative contribution between optimization proposed solution and experimental trials, where empirical continuous improvement techniques may prove beneficial.

\section{References}

1. Antonelli, D., Bruno, G.: Dynamic distribution of assembly tasks in a collaborative workcell of humans and robots. FME Transactions 47(4), 723-730 (2019)

2. Battini, D., Calzavara, M., Otto, A., Sgarbossa, F.: Preventing ergonomic risks with integrated planning on assembly line balancing and parts feeding. International Journal of Production Research 55(24), 7452-7472 (2017)

3. Battini, D., Delorme, X., Dolgui, A., Persona, A., Sgarbossa, F.: Ergonomics in assembly line balancing based on energy expenditure: a multi-objective model. International Journal of Production Research 54(3), 824-845 (2016)

4. Bilberg, A., Malik, A.A.: Digital twin driven human-robot collaborative assembly. CIRP Annals 68(1), 499-502 (2019)

5. Malik, A.A., Bilberg, A.: Collaborative robots in assembly: A practical approach for tasks distribution. In: 52nd CIRP Conference on Manufacturing Systems. vol. 81, pp. 665-670 (2019)

6. McAtamney, L., Corlett, E.N.: Rula: a survey method for the investigation of workrelated upper limb disorders. Applied ergonomics 24(2), 91-99 (1993)

7. Pearce, M., Mutlu, B., Shah, J., Radwin, R.: Optimizing makespan and ergonomics in integrating collaborative robots into manufacturing processes. IEEE transactions on automation science and engineering 15(4), 1772-1784 (2018)

8. Quenehen, A., Pocachard, J., Klement, N.: Process optimisation using collaborative robots-comparative case study. IFAC-PapersOnLine 52(13), 60-65 (2019)

9. Thiemermann, S.: Direkte Mensch-Roboter-Kooperation in der Kleinteilemontage mit einem SCARA-Roboter (2005)

10. Tsarouchi, P., Matthaiakis, A.S., Makris, S., Chryssolouris, G.: On a human-robot collaboration in an assembly cell. International Journal of Computer Integrated Manufacturing 30(6), 580-589 (2017)

11. Weckenborg, C., Spengler, T.S.: Assembly line balancing with collaborative robots under consideration of ergonomics: a cost-oriented approach. IFAC-PapersOnLine 52(13), 1860-1865 (2019) 